\title{
Elasticidades de demanda de un medicamento antidepresivo en Colombia como estrategia para evaluar poder de mercado*
}

\section{Elasticity of Demand of an Antidepressant Drug in Colombia as an Strategy to Assess Market Power}

\section{Elasticidades de demanda de um medicamento antidepressivo na Colômbia como estratégia para avaliar o poder de mercado}

Fecha de recepción: 05-04-16 Fecha de aceptación: 02-11-16 Disponible en línea: 30-11-16 doi:10.11144/Javeriana.rgyps15-31.edma

Cómo citar este artículo:

Cadena-Lozano JB, Ariza-Garzón MJ, Pulido-Cruz CG. Elasticidades de demanda de un medicamento antidepresivo en Colombia como estrategia para evaluar poder de mercado. Rev. Gerenc. Polít. Salud. 2016; 15(31): 278-297. http://dx.doi.org/10.11144/Javeriana.rgyps15-31.edma

\author{
Javier Bernardo Cadena-Lozano ** \\ Miller Janny Ariza-Garzón *** \\ Carlos Gerardo Pulido-Cruz $* * * *$
}

Artículo de investigación producto de la línea de Finanzas y Economía del Grupo de Estudios en Administración (GEA) del Colegio de Estudios Superiores de Administración (CESA) y de la línea de Gestión del Riesgo y Optimización del Grupo Innovatic de la Universidad Piloto de Colombia.

** Doctor en Administración de la Universidad San Pablo Ceu, Madrid, España. Magíster en Economía de la Universidad Nacional de Colombia, Bogotá, Colombia. Magíster en Dirección y Desarrollo de Empresas de la Universidad de Ávila, España. Especialista en Finanzas Privadas de la Universidad del Rosario, Bogotá, Colombia. Economista de la Universidad del Rosario, Bogotá, Colombia. Profesor asociado del Colegio de Estudios Superiores de Administración (CESA), Bogotá, Colombia. Dirección oficina: Calle 35 N.o 5A-31. Correo electrónico: javier.cadena@cesa.edu.co

*** Magíster en Economía de la Universidad Javeriana, Bogotá, Colombia. Estadístico de la Universidad Nacional de Colombia, Bogotá, Colombia. Licenciado en Matemáticas de la Universidad Distrital Francisco José de Caldas, Bogotá, Colombia. Profesor-investigador del Programa de Ingeniería Financiera de la Universidad Piloto, Bogotá,

278 Colombia. Correo electrónico: miller-ariza@unipiloto.edu.co Contador público de la Universidad Piloto, Bogotá, Colombia. Correo electrónico: cgpc1984@yahoo.es 


\section{Resumo}

En este artículo se desarrolló un ejercicio de estimación de la elasticidad precio de la demanda de un medicamento "tipo" utilizado para el tratamiento de la depresión. Este trastorno afecta física y mentalmente a un gran número de personas en el mundo y constituye un problema de salud pública con prevalencia creciente. Se estimaron dos modelos econométricos vectoriales para el periodo anterior a la expedición de la circular 03 del 2013 que regula los precios de los medicamentos en Colombia. Como resultado se obtuvo una elasticidad precio no significativa de $0.35 \%$, es decir, que los cambios en el precio no generan la respuesta esperada en la demanda, comportamiento que sugiere la existencia de un alto poder de mercado de la empresa que elabora dicho producto "tipo", lo cual es evidencia de la necesidad de implementar dicha circular.

Palavras-chave: antidepresivos; preparaciones farmacéuticas; elasticidad; política de salud; política social

\section{Abstract}

This article develops an estimation of the price elasticity of demand of a medication "type" for treating depression. This disorder affects physically and mentally a big part of the population around the world. This disorder is considered an important public health problem whose prevalence has been increasing. For the purpose of this research two econometric models of vectors were estimated for the previous period of the issuance of Circular 03 of 2013, which regulates the price of medications in Colombia. The outcomes of the estimation show a non-significant price elasticity of $0.35 \%$, that is to say, changes in price do not generate the expected response in demand. This result suggests that the company, which produces this type of medication, exerts significant power in the market. Therefore, this result proves the need to implement the Circular.

Keywords: antidepressive agents; pharmaceutical preparations; elasticity; health policy; public policy.

\section{Resumen}

Neste artigo é desenvolvido um exercício de estimação da elasticidade-preço da demanda de um medicamento "tipo" utilizado para tratamento da depressão. Tal transtorno afeta física e mentalmente um grande número de pessoas no mundo e constitui um problema de saúde pública com prevalência crescente. Estimaram-se dois modelos econométricos vetoriais para o período anterior à emissão do edital 03 de 2013 que regula os preços dos medicamentos na Colômbia. Como resultado, foi obtida uma elasticidade-preço não significativa de $0.35 \%$, ou seja, que as mudanças no preço não geram a resposta esperada na demanda, comportamento que sugere a existência de alto poder de mercado da empresa que elabora tal produto "tipo", evidenciando a necessidade de implementar o edital.

Palabras clave: antidepressivos; preparações farmacêuticas; elasticidade; política de saúde; política social 


\section{Introducción}

La estimación de las elasticidades de demanda de un medicamento esencial para el tratamiento de cualquier patología es de gran importancia para el conocimiento del mercado y para los reguladores de precios del sector. La identificación de las variables y su grado de sensibilidad ofrece alternativas para la formulación de una política pública que regule el comportamiento del mercado farmacéutico en Colombia.

Dentro de las enfermedades con mayor prevalencia en Colombia se encuentra la depresión; este trastorno es uno de los retos del Sistema de Seguridad en Salud tanto para su detección como para su tratamiento. Por esta razón y dado el tamaño de mercado y su complejidad, en este artículo se presenta un ejercicio de estimación de la elasticidad precio de la demanda de un medicamento "tipo" producido por un laboratorio de marca utilizado en el tratamiento de la depresión.

El objetivo consistió en determinar si desde el primer trimestre del 2007, periodo anterior a la expedición de la Circular 03 del 2013, donde predominó la libertad de precios y se configuró un proceso de aumento en el precio de los productos originales de las grandes multinacionales, existió poder de mercado.

Para la estimación de las elasticidades se especifican dos modelos de vectores autorregresivos (VAR) y VAR con corrección del error. Con base en sus resultados, junto con otro tipo de análisis, fue posible establecer comportamientos monopólicos de estas empresas que sustentan la necesidad de establecer una política de control en este frente, como se evidenció mediante la Circular 03 del 2013 de la Comisión Nacional de Precios de Medicamentos y Dispositivos Médicos (CNPMDM).

\section{El mercado farmacéutico y de antidepresivos colombiano}

De acuerdo con el documento Conpes ${ }^{1}$ Social 155 del 30 de agosto del 2012 (1), que resume la situación del sector farmacéutico, es posible identificar a partir de la primera década del presente siglo tres momentos en la regulación previos a la Resolución 03 del 2013 de la CNPMDM: hasta el primer trimestre del 2002, caracterizado por una intervención amplia con procedimientos automáticos de cambio de régimen de regulación y definición de precios de venta al público; un segundo periodo (2002- 2006) con menor intervención que fue flexibilizando el régimen de control de medicamentos; y un tercer periodo, de libertad de precios con algunas excepciones a partir del 2006. De acuerdo con Vásquez, Gómez y Rodríguez (2) mediante la circular 004 del 2006 se estableció que todos los medicamentos comercializados en Colombia, con algunas excepciones, se incluirían en el régimen de libertad vigilada; entre el 2006 y el 2010 la variación del IPC de medicamentos se ubicó entre el $11 \mathrm{y}$ el $18 \%$, siempre superior a la inflación del sector salud y general.

Según dicho documento, cuando se analizan precios de medicamentos pioneros sin competencia, diversas investigaciones registran valores que en general están por encima a los observados en países pares tomados como referencia. Como ejemplo, comparan seis medicamentos monopólicos de alto recobro y encuentran que en cuatro casos el precio relativo en Colombia es superior al promedio observado en América Latina en un rango entre 27 y $140 \%$. Comparan también ocho medicamentos oligopólicos donde cinco referencias presentan precios relativos superiores al promedio de América Latina, en rangos que varían entre 2 y $60 \%$.

1 Consejo Nacional de Política Económica y Social. 
Tabla 1. Mercado farmacéutico Colombiano 2011

\begin{tabular}{|l|c|c|c|c|}
\hline \multicolumn{5}{|c|}{ Tamaño del mercado farmacéutico por diversas fuentes y eslabones de la cadena de comercialización } \\
& \multicolumn{4}{c|}{ Fuente } \\
\hline 2011 & $\begin{array}{c}\text { IMS/farmacia } \\
\text { (millones de } \\
\text { \$CP) }\end{array}$ & $\begin{array}{c}\text { IMS (millones } \\
\text { de US) }\end{array}$ & $\begin{array}{c}\text { Sismed venta } \\
\text { laboratorios (mil- } \\
\text { lones de \$CP) }\end{array}$ & $\begin{array}{c}\text { Sismed venta } \\
\text { laboratorios } \\
\text { (millones de US) }\end{array}$ \\
\hline $\begin{array}{l}\text { Total mercado } \\
\text { farmacéutico (según } \\
\text { canales) }\end{array}$ & 5940000.00 & 3300.00 & 5080554.36 & 2822.53 \\
\hline Comercial & 3960000.00 & $2200.00 *$ & 3215301.00 & $1786.28^{* *}$ \\
\hline Institucional & 1980000.00 & 1100.00 a & 1865253.36 & $1036.25^{* *}$ \\
\hline
\end{tabular}

Tasa de cambio promedio $\$ 1800$

* Dato observado

a Estimado (institucional: 50\% del total según IMS)

** Dato observado Sismed

Fuente: tomado de (4)

Con estos antecedentes conocidos por la CNPMDM, esta expide el 21 de mayo la Circular 03 del 2013, "por la cual se establece la metodología para la aplicación del régimen de control directo de precios para los medicamentos que se comercialicen en el territorio nacional" (3). Las modalidades de control que se fijan son dos: un régimen de libertad vigilada y un régimen de control directo.

En el primero, quienes vendan medicamentos podrán establecer sus precios libremente. En el segundo, teniendo en cuenta un periodo, la Comisión fija el precio máximo en uno o varios niveles de la cadena de comercialización; en ambos casos las empresas deberán informar a la autoridad competente sobre sus transacciones comerciales, de acuerdo con la normatividad vigente. Si bien esta medida fue bien recibida por la mayoría de los agentes del mercado, es necesario esperar a consolidar las nuevas cifras y evaluar la efectividad de esta política nacional.

En cuanto a la situación del mercado farmacéutico previamente a la Circular 03 del 2013, el documento Conpes (proyecto para discusión) advierte sobre los problemas de información en términos de precios, uso y calidad de los medicamentos: esta es escasa, limitada y poco estandarizada, situación que "constituye una importante limitación para la toma de decisiones y el monitoreo a los resultados en salud" (4).

En este contexto, no se conoce con certeza el valor de mercado del sector farmacéutico. En efecto,

Según (IMS ${ }^{2}$ ), en 2011 el mercado privado en Colombia alcanzó los US\$2,2 mil millones, al cual debe agregarse el valor del mercado institucional (que la firma estima en cerca del $50 \%$ del mercado comercial), para un total de US\$ 3,3 mil millones. Pero la estimación de SISMED $^{3}$, tomando el registro de ventas de los laboratorios farmacéuticos, asciende a US\$ 2,8 mil millones. (4) (Véase tabla 1)

2 IMS-Health es una entidad especializada en llevar estadísticas del sector farmacéutico a nivel global

3 Sismed es el Sistema de Información de Precios de Medicamentos que se constituye como una herramienta de control, administración y análisis de los precios de los medicamentos en Colombia. Es controlado por la Comisión Nacional de Precios de Medicamentos (CNPM) y a cargo del Ministerio de la Protección Social y del Ministerio de Comercio, Industria y Turismo. 
Con respecto a la depresión, Machado, Morales y Solarte (5) la definen como un trastorno que afecta al individuo en su funcionamiento biológico, psicológico y social y se constituye en un problema de salud pública con prevalencia creciente. Las formas más comunes en que se presenta son el trastorno depresivo mayor, la distimia ${ }^{4}$ y la fase depresiva del trastorno bipolar.

Los autores en mención señalan que la prevalencia en Colombia ha variado entre 12,1 y $19,6 \%$ y es más común en las mujeres. De acuerdo con estos indicadores, uno de los retos del Sistema de Seguridad Social en Salud es el mejoramiento de la detección y el acceso al tratamiento de la depresión, que si bien en este sistema solo se tiene acceso a los productos incluidos en el Plan Obligatorio de Salud (POS), la mayoría de productos fabricados y comercializados por las grandes casas farmacéuticas deben ser adquiridos fuera del sistema, ya sea que los prescriptores realicen la solicitud a las instituciones prestadoras de servicios de salud a través de los comités técnico científicos, o ya sea que el paciente compre el medicamento en el mercado comercial.

El mercado de antidepresivos en Colombia para el canal comercial es un mercado con ventas en 2011 de $\$ 33593$ millones y crecimiento compuesto ${ }^{5}$ entre el 2007 y el 2011 de $7,9 \%$.

El mercado está conformado por marcas originales, genéricos de marca (BGx) y genéricos $(\mathrm{Gx})$. La participación en ventas

4 La distimia es un trastorno afectivo crónico caracterizado por una bajo autoestima, estado de ánimo melancólico y tristeza y su origen es de tipo genético-hereditario.

5 El crecimiento compuesto, en sus siglas en inglés CAGR (Compound Annual Growth Rate), es un método de estimación del promedio de crecimiento porcentual anual, en este caso de las ventas y unidades estándar en un periodo específico: 2007 2011. CAGR $\left.=\frac{\text { Valor final }}{\text { Valor inicial }} \frac{1}{\text { nde años }}\right)-1$ de cada tipo de producto en el año 2007 fue en su orden de 66,22 y $12 \%$, con un repunte importante de los genéricos en el 2011 con participaciones de $53 \%$ para los originales, $31 \%$ para los BGx y $16 \%$ para los Gx. El crecimiento compuesto de estos mismos productos en el periodo 2007-2011 fue de $2 \%{ }^{6}$ para los originales, $18 \%$ para los BGxy $15,6 \%$ para los Gx.

Si bien en valores los productos originales crecen —por ejemplo, en el 2011 con un $0,7 \%$ - las unidades vendidas muestran un decrecimiento del $0,5 \%$, con lo cual se infiere que el crecimiento obtenido en valores, en general y para todo el periodo 2007-2011 viene dado por incrementos de precio. En el caso de los BGx y los Gx el crecimiento es explicado por su mayor volumen y precios, como por ejemplo en el 2011, donde el crecimiento en valores de los BGx fue de 17,3\% y en unidades de $11,7 \%$.

El mercado de antidepresivos está conformado por seis moléculas. El laboratorio de análisis participa con el $26 \%$ y el producto "tipo" con el $50 \%$ de dicho porcentaje.

\section{Funciones de demanda de medicamentos}

La importancia de estimar funciones de demanda para conocer la sensibilidad de cada una de sus variables ha sido tema de estudio en la mayoría de sectores de la economía, con el fin de soportar decisiones gubernamentales de control y en el ámbito de las empresas, en términos financieros y de marketing. En particular, en el sector farmacéutico se encuentra una mayor investigación en temas de pronóstico de la demanda, en contraste con la estimación de sus elasticidades.

6 En términos de volumen se presentó un decrecimiento en el periodo $2007-2011$ del $-4,0 \%$. 
En este sentido, el Center for Global Development (6) sugiere un set de once técnicas de pronóstico para medicamentos, las cuales se pueden utilizar dependiendo del ciclo de vida del producto y de las condiciones del mercado; estas metodologías abarcan desde el conocimiento de expertos hasta el uso de técnicas cuantitativas como la extrapolación y los modelos causales.

Según la Managment Science for Health (7), los métodos para estimar las necesidades de medicamentos para el consumo sirven como herramienta para preparar y justificar los presupuestos farmacéuticos, planear el lanzamiento de nuevos productos, mejorar la asignación del presupuesto basado en las prioridades del sistema de salud, mejorar la efectividad de los tratamientos, calcular las necesidades en desastres o epidemias, estimar el reabastecimiento de productos que se agotan con mayor frecuencia y comparar el consumo de medicamentos con las prioridades públicas del sistema de salud.

Para Ghousi, Mehrani, Momeni y Anjomshoaa (8), el pronóstico desempeña un papel trascendental para mejorar la ventaja competitiva, así como la planificación de la producción y el control de inventarios. Los autores, a través de técnicas como la minería de datos, y específicamente algoritmos de reglas de asociación, predicen el comportamiento futuro del consumo de medicamentos. Así, comparan tres técnicas diferentes: regresión, redes neuronales y árboles de decisión, con el propósito de elegir la técnica con mayor precisión para seis tipos de medicamentos que corresponden a seis diagnósticos diferentes. Toman una muestra de tres años, con cerca de 407000 registros obtenidos de un distribuidor en Irán, donde variables asociadas a la distribución de los medicamentos y las características de la población son importantes para predecir el consumo. La investigación concluye que la técnica de pronóstico más precisa es la de árboles de decisión, excepto cuando el modelo se utiliza para el pronóstico de medicamentos para tratar el asma; para este tipo de medicamentos la técnica que presenta el mejor ajuste es la de redes neuronales.

Ching (9), motivado por la baja difusión de medicamentos genéricos y el comportamiento de los precios de los medicamentos de marca, estimó un modelo de prescripción de drogas - Modelo de Demanda Aprendizaje Bayesiano - el cual incorpora el aprendizaje y la heterogeneidad de los consumidores. Infiere que el público es adverso al riesgo, dudoso de la calidad de los medicamentos genéricos y tiene una predisposición pesimista. En contraste con estudios previos, Ching encontró que la elasticidad precio de la demanda de los medicamentos de marca se incrementa con el tiempo y tiende a ser inelástica (menor que uno). Los resultados demuestran que los laboratorios de marca, en un horizonte de largo plazo, establecen precios más bajos que los que ellos fijarían en un horizonte de corto plazo, ya que al hacer esto reducen la velocidad del proceso de aprendizaje para los genéricos. Pero tal incentivo disminuye en el tiempo, en la medida en que las dudas de los genéricos se resuelven y los laboratorios de marca inician una fase gradual de incremento en precios.

Kim, Won, Park y Kang (10) desarrollan dos modelos que capturan los efectos que tiene la información y el análisis trend topic (tendencias del momento) de las redes sociales para cuatro medicamentos usados para la hipertensión, la úlcera, la próstata y enfermedades de los ojos. El objetivo es pronosticar la demanda de medicamentos a través del modelamiento de las tendencias temáticas de blogs en la web. La muestra es de 44 meses, entre enero del 2010 y agosto del 2013, y se utilizan dos variables: una serie de tiempo de ventas y otra que con- 
tiene trend topic del medicamento utilizado. Desarrollan dos modelos tipo VARX (vector autorregrresivo con variables exógenas), un modelo autorregresivo con la variable endógena ventas, y un modelo que además de incluir la variable endógena, incluye la variable exógena denominada media móvil del trend topic. Los autores concluyen que el pronóstico tiene un mejor desempeño cuando se incluye la variable exógena, que la demanda por los medicamentos para la hipertensión y la úlcera no está afectada por el comportamiento social media y que la bondad del ajuste del pronóstico de los medicamentos para la próstata y enfermedades de los ojos mejora significativamente cuando se introduce la variable de trend topic, con una reducción promedio del error de pronóstico de 7\%. Esta investigación tiene un gran aporte teórico y práctico, pues genera conocimiento acerca de la inclusión en los modelos cuantitativos de variables contenidas a través de medios web que capturan la aceptación de un producto por parte del mercado.

En cuanto a la estimación especifica de las elasticidades, Fisher, Cockburn, Griliches y Hausman (11) proponen un modelo de demanda para cuatro cefalosporinas (antibiótico de espectro amplio); calculan la elasticidad precio de la demanda y la elasticidad cruzada entre los productos de marca y genéricos. Utilizan un modelo multietapa con el supuesto de que este es apropiado para el funcionamiento de las compras de productos farmacéuticos, en particular en las etapas de prescripción y dispensación.

Las series que utilizan son mensuales - de octubre de 1985 a marzo de 1991 - de las cantidades y ventas a los mayoristas por presentación separada entre hospitales y droguerías. Se focalizan en el mercado de 284 droguerías, por ser el más grande e importante para este tipo de productos. Del análisis del modelo concluyen que existen altas elasticidades entre genéricos sustitutos y entre algunas terapias sustitutas, es decir, el consumidor se mueve a través de un árbol de decisión; su elección parece estar afectada por las diferencias de precios cuando selecciona entre dos medicamentos para la misma terapia; una vez ha tomado la decisión de la terapia, las diferencias de precios parecen ser muy importantes cuando selecciona entre marca y genérico.

Cabe resaltar que dentro de la simulación de los modelos, los autores encontraron elasticidades precio y cruzada para el medicamento 1 (cefalexina) no significativas y con un signo no esperado; por el contrario, para el medicamento 3 (cefadina) las elasticidades fueron significativas y con el signo esperado.

\section{Materiales y métodos}

El estudio desarrollado es del tipo descriptivo correlacional, pues busca describir y estimar la múltiple interdependencia que puede existir entre las diferentes variables de un sistema de demanda.

El periodo analizado con información mensual abarca cinco años previos a la Circular 03 del 2013, expedida por el Ministerio de Salud, con el objetivo de regular los precios de los medicamentos. En una primera fase se consideran nueve variables, las cuales se detallan a continuación: ${ }^{7}$

- Cantidades demandadas per cápita (QP_TIPO): se utiliza la información reportada por IMS Colombia; esta suministra las ventas en valores y unidades estándar de los productos antidepresivos. Las unidades estándar per cápita se ob-

7 Las variables del mercado de antidepresivos colombiano se tomaron a precios constantes del 2005. 
tienen de dividir las unidades estándar por la población proyectada según el Departamento Administrativo Nacional de Estadística (DANE) (años 2005-2020).

- Precio medicamento evaluado ( $\mathrm{P}_{-}$ TIPO): el precio del medicamento es calculado de la división del valor total de las ventas sobre las unidades estándar per cápita, información suministrada por IMS Colombia.

- $\quad$ Precio de los medicamentos sustitutos (P_ORIG; P_BGX; P_GX): de la misma manera como se estimó el precio del medicamento evaluado, se calcularon los precios para los medicamentos sustitutos de tipo original (P_ORIG), genéricos de marca (P_BGX) y genéricos (P_GX), los dos primeros producidos por casas farmacéuticas y el último por empresas nacionales.

- Índice de precios al productor (IPPT): de acuerdo con la Circular 004 del 2006, artículo 9. , parágrafo $3 .^{\circ}$ de la CNPMDM, el incremento de los medicamentos que estén en libertad regulada no debe superar el crecimiento del IPPT; por tal razón es fundamental como variable que explica el incremento en el precio de los medicamentos. La información se tomó del DANE.

- $\quad$ Tasa representativa del mercado (TRM): todos los productos del laboratorio que produce el medicamento "tipo" son importados y se ven muy afectados por la apreciación o depreciación del peso colombiano con respecto al dólar. Estas cifras se tomaron con fuente del Banco de la República.

- Ingreso de los hogares (ING_HOG): información suministrada por la firma
Raddar Colombia, empresa que hace mediciones del consumo de los hogares. ${ }^{8}$

- Producto Interno Bruto (PIB): corresponde a la serie desestacionalizada en forma trimestral. Información con fuente DANE.

Dado que no existe una estimación oficial de la variable ingreso como serie temporal, se utiliza como variable proxy de esta el PIB, el cual se calcula en forma trimestral; se quiso dejar esta variable junto con el ingreso mensual de los hogares estimado por la firma Raddar, y mediante pruebas econométricas analizar cuál de las dos ofrecía una mejor estimación y validación del modelo. La diferencia en periodicidad hizo necesario utilizar un modelo de desagregación temporal de series, con el fin de obtener la información del PIB mensual.

Por tal razón, se analizaron diferentes modelos o métodos univariados de desagregación temporal como los de Denton, Chow y Lin, Fernández y Litterman, métodos cuya descripción detallada es presentada por Hurtado y Melo (12) en su artículo sobre desagregación temporal. En todos los casos se busca generar una serie del PIB mensual (alta frecuencia o periodicidad) a partir del PIB trimestral (baja frecuencia).

Para la desagregación del PIB según los métodos anteriores, se tomó como base los desarrollos de Quilis (13). Dicha biblioteca se analizó y adaptó para estimar los valores mensuales del PIB para el periodo 2007-2011.

De los resultados de cada estimación solo se muestran los dos criterios de información propuestos por Quilis, que son el de Akaike

8 Más adelante se analizan otras variables proxy del ingreso. 
Tabla 2. Valores de los CRITERIos de información Para los MÉTOdos de desagregación del PIB

\begin{tabular}{|l|c|c|}
\hline Método & AIC & BIC \\
\hline Chow y Lin & 13.90 & 14.05 \\
\hline Fernández & 14.23 & 14.33 \\
\hline
\end{tabular}

Fuente: elaboración propia

(AIC) ${ }^{9}$ y el de Schwartz (BIC). ${ }^{10}$ De acuerdo con la tabla 2 se escogió el PIB mensual estimado por el método de Chow y Lin, debido a que los indicadores AIC y BIC tienen el menor valor. Es importante mencionar que esta metodología sugiere el uso de variables que contribuyan a mejorar las estimaciones; en este caso se utilizaron las variables Imaco $^{11} \mathrm{e}$ índice mensual manufacturero del DANE. En adelante el PIB resultante se denominada "PIBCH".

Definida la serie PIBCH como variable proxy del ingreso - como opción alternativa a la serie ingreso de los hogares (ING_HOG) a continuación se describe el procedimiento seguido para la especificación, la estimación y la validación de dos modelos, donde el objetivo principal es el cálculo de la elasticidad precio de la demanda, aunque también se muestran las estimaciones de las elasticidades ingreso y cruzada de la demanda.

En la revisión de la literatura se encontró que en el análisis de las funciones de demanda estimadas es posible trabajar con modelos uniecuacionales y multiecuacionales. Sin embargo, como lo señalan Flores y Pereira (14)

9 AIC: es un criterio de selección de modelos que se basa en la teoría de información; es útil para evaluar el desempeño de la predicción dentro y fuera de la muestra. Su expresión es: $\ln \left(\frac{S C E}{T-K}\right)+2 \frac{K}{T}$, donde $S C E$ es la suma de cuadrados del error, $T$ el tamaño muestral y $k$ el número de regresores usados en el modelo.

10 BIC: es un criterio alternativo de selección de modelos con un enfoque bayesiano. Su expresión es $\ln \left(\frac{S C E}{T-K}\right)+\frac{K}{T} \ln T$

11 Es un indicador mensual construido por el Banco de la República a partir de variables sectoriales que anticipa con cinco meses de adelanto los movimientos del crecimiento anual del PIB acumulado cuatro trimestres. y Roca y Pereira (15), cuando el propósito consiste en estimar una elasticidad no es adecuado utilizar modelos uniecuacionales. Según estos autores, dichos enfoques excluyen la probable presencia de efectos de retroalimentación o feedback entre las variables consideradas, es decir, no recogen los múltiples efectos que en todas direcciones se pueden producir a lo largo del tiempo entre las diversas variables relevantes del modelo.

En este contexto, se decidió por la especificación de un modelo de vectores autorregresivos (VAR) y un VAR con corrección del error, los cuales tienen la ventaja de ser dinámicos en cuanto que un cambio en la perturbación de cualquiera de las ecuaciones del modelo, además de impactar la variable dependiente en la misma ecuación, afecta la totalidad de variables del modelo por varios periodos, vía los rezagos de la variable de impulso en los demás componentes del sistema.

Los modelos VAR fueron propuestos por Sims en 1980 y han sido utilizados para predecir sistemas de series temporales interrelacionadas y para analizar la dinámica que se genera en el sistema ante el impacto de un choque aleatorio en alguna de las variables que lo conforma. Para esto se utiliza la función de impulso respuesta generalizada (GFIR) propuesta por Pesaran y Shin (16), la cual permite que el ordenamiento de las variables no afecte los resultados ni la interpretación adecuada de dicha función. El modelo de corrección del error se considera un modelo VAR restringido, diseñado para ser utilizado con series que son no estacionarias, pero que están cointegradas; dicho mod- 
elo recoge información importante sobre las relaciones de equilibrio a largo plazo entre las variables e identifica los desequilibrios que existen en el corto plazo.

\section{Resultados}

Para la especificación de los modelos VAR propuestos, en el anexo 1 se presentan las pruebas de estacionariedad requeridas; en el anexo 2 se muestra el proceso para la selección de la variable proxy del ingreso, a partir de la estimación de dos modelos VAR. Se concluye que el PIB con el método de desagregación de Chow y Lin (PIBCH) presenta mejores resultados, según el criterio de Akaike.

La estimación de los parámetros del modelo VAR se detalla en la tabla 3. La variable endógena corresponde a la demanda per cápita del producto "tipo" (QP_TIPO). El modelo con el total de variables se presenta en el anexo 3.

La validación del modelo, que implica realizar las pruebas de ruido blanco sobre los residuos y de estabilidad sobre los inversos de las raíces de los polinomios de rezago asociados se resumen en el anexo 4.
A partir de las funciones de impulso-respuesta se estima la elasticidad precio de la demanda del producto "tipo" por periodo y acumulada. Esta última tiene en cuenta los efectos acumulados a largo plazo en la cantidad demandada ante un choque temporal en el precio; es decir, muestra el resultado de acumular la respuesta que tendrá la demanda ante un impulso durante varios periodos: indica en qué porcentaje ha cambiado la cantidad demandada del producto debido a un cambio transitorio en su precio, teniendo en cuenta no solo el primer periodo, sino todos aquellos en los cuales la cantidad demandada ha cambiado por efecto del choque.

En la tabla 4 se muestran los valores de cambio mensual en la demanda del producto "tipo" de " $\mathrm{t}$ " a " $\mathrm{t}+5$ " ante una innovación en su precio. La tabla completa con todas las variables del sistema se presenta en el anexo 5 .

En la figura 1 se muestran las funciones de impulso-respuesta para las variables QP_TIPO y P_TIPO.

Allí se observa cómo un choque positivo del P_TIPO sobre sí mismo en el periodo t es de 0.034; este mismo sobre QP_TIPO produce un aumento en el periodo $\mathrm{t}$ (instantáneo) de 0.012 , que equivale a un incremento del

Tabla 3. Coeficientes estimados del modelo VAR PARA la demanda del PRoducto "Tipo"

\begin{tabular}{|l|c|c|}
\hline Variables & Coeficientes & t-estadísticos \\
\hline QP_TIPO(-1) & 0.51 & 4.53 \\
\hline P_TIPO(-1) & 1.11 & 2.39 \\
\hline P_BGX(-1) & 0.07 & 0.19 \\
\hline D(P_GX(-1)) & -0.55 & -1.14 \\
\hline P_ORIG(-1) & $-0,31$ & $-0,89$ \\
\hline D(PIBPC_CH(-1)) & -0.21 & -0.36 \\
\hline D(IPPT(-1)) & -0.01 & -0.21 \\
\hline D(TRM(-1)) & -0.01 & -0.02 \\
\hline C & -5.10 & -1.92 \\
\hline
\end{tabular}

Fuente: elaboración propia 
Tabla 4. Respuesta de QP_TIPO al impulso de P_TIPO

\begin{tabular}{|l|c|}
\hline Periodo & Respuesta \\
\hline $\mathrm{t}$ & 0.012 \\
\hline $\mathrm{t}+1$ & 0.030 \\
\hline $\mathrm{t}+2$ & $\mathbf{0 . 0 4 4}$ \\
\hline $\mathrm{t}+3$ & $\mathbf{0 . 0 3 0}$ \\
\hline $\mathrm{t}+4$ & $\mathbf{0 . 0 3 0}$ \\
\hline $\mathrm{t}+5$ & $\mathbf{0 . 0 2 3}$ \\
\hline
\end{tabular}

Fuente: elaboración propia

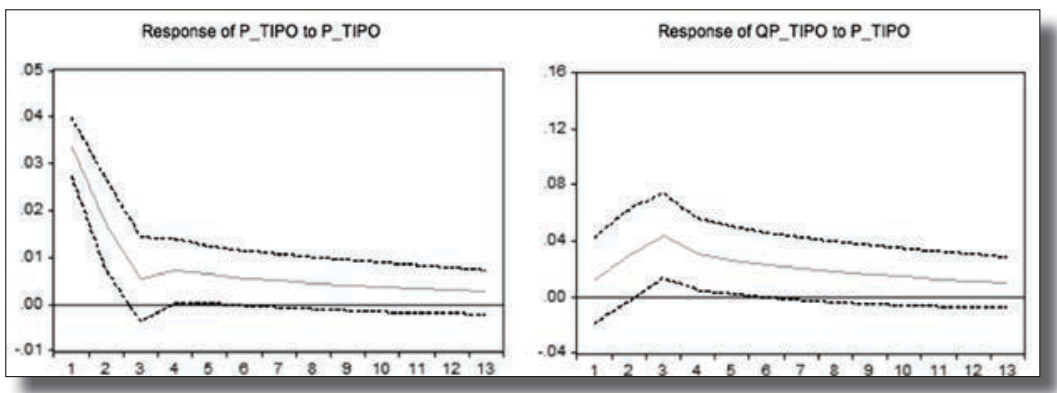

Figura 1. Respuesta al impulso de las variables QP_TIPO y P_TIPO

Fuente: elaboración propia

$0.35 \%$ ante un aumento del precio del $1 \%$; en $t+1$ de 0.03 , y solo significativo entre los periodos $\mathrm{t}+2(0.044) \mathrm{y} t+5(0.023)$ inclusive, donde los límites de confianza se encuentran por encima de cero (véase figura 1). Como se evidencia, el signo de la elasticidad precio de la demanda no corresponde con la teoría, es decir, que en este caso se establece una relación directa entre precio y cantidad del producto "tipo".

Para analizar con mayor detalle el resultado anterior, y dado que algunas variables que se incluyeron en el modelo VAR son integradas de orden uno, se estimó un modelo de corrección del error para el cálculo de las elasticidades.

El modelo escogido para la ecuación de cointegración corresponde a la opción sin tendencia determinista, en niveles y sin intercepto. En el anexo 6 se muestran los valores de probabilidad para las distintas opciones.
En dicho modelo los coeficientes del vector de cointegración estimado para las variables P_GX, IPPT, TRM y PIBPC_CH son, en su orden:

$\beta^{\prime}=\left(\begin{array}{lll}1.00000 & -0.0935692 .091971-0.780726\end{array}\right)$

Estos se utilizaron para la estimación del modelo VAR con corrección del error (véase anexo 7). El test de validación de Portmanteau y la gráfica de los inversos de las raíces del polinomio característico asociado se encuentran en el anexo 8.

El análisis de la función de impulso-respuesta muestra que un choque positivo del P_TIPO sobre sí mismo en el periodo t es de 0.0336 ; de este sobre QP_TIPO produce un aumento en el periodo t no significativo de 0.0078 ; es muy poco significativo y positivo en el periodo $t+2$, y despreciable en términos estadísticos en todo el periodo de análisis (véanse tabla 5 y figura 2). Lo anterior implica que 
no hay respuesta significativa por parte de la demanda ante cambios en el precio; este comportamiento sugiere la existencia de un alto poder de mercado de la empresa que elabora el producto "tipo".

En conclusión, un choque en el precio en el periodo $t$ solo se refleja en la cantidad en $t+2$, en tanto que se diluye en periodos posteriores. Un efecto muy pequeño e inferior sobre la cantidad del producto "tipo" tiene un choque en el precio del sustituto genérico de marca importado (en $\mathrm{t}+2$ de 0.0256). Por su parte, la respuesta de la demanda del producto "tipo" ante un impulso de las variables precio de los demás genéricos y del ingreso no evidencia un cambio significativo en ningún periodo; es decir, no afectan su comportamiento.

\section{Discusión y conclusiones}

Los modelos multivariados desarrollados muestran para la demanda del producto "tipo" una total insensibilidad al precio que confirma tendencias y conclusiones de otros estudios, en el sentido de que en el sector de medicamentos se pueden encontrar elasticidades negativas muy bajas, cercanas a cero e incluso positivas. Este hallazgo sugiere la posibilidad de que algunos laboratorios de marca en Colombia hayan tenido alto poder de mercado, situación que sustenta la expedición de la Circular 03 del 2013 que regula los precios de los medicamentos.

Estudios como los de Torres y Gutiérrez (17) y Callejón y Ortún (18) llegan a las mismas conclusiones. Los primeros estimaron la elasticidad precio de la demanda de los productos farmacéuticos en México, mediante el análisis de un producto índice para los años 2003 (0.007), 2004 (0.003) у 2005 (-0.002). Los datos en paréntesis corresponden a las elasticidades, las cuales en general tienden a cero en todos los casos.

Tabla 5. Respuesta de QP_TIPO a diferentes impulsos

\begin{tabular}{|c|c|c|}
\hline \multirow{2}{*}{ Periodo } & \multicolumn{2}{|c|}{ Variables de impulso } \\
\hline & P_TIPO & P_BGX \\
\hline $\mathrm{t}$ & 0.0078 & 0.0079 \\
\hline$t+1$ & 0.0209 & 0.0154 \\
\hline$t+2$ & 0.0320 & 0.0256 \\
\hline$t+3$ & 0.0175 & 0.0170 \\
\hline$t+4$ & 0.0143 & 0.0162 \\
\hline$t+5$ & 0.0119 & 0.0143 \\
\hline$t+6$ & 0.0103 & 0.0125 \\
\hline$t+7$ & 0.0088 & 0.0107 \\
\hline$t+8$ & 0.0077 & 0.0092 \\
\hline$t+9$ & 0.0066 & 0.0079 \\
\hline$t+10$ & 0.0056 & 0.0067 \\
\hline$t+11$ & 0.0048 & 0.0058 \\
\hline$t+12$ & 0.0041 & 0.0049 \\
\hline
\end{tabular}




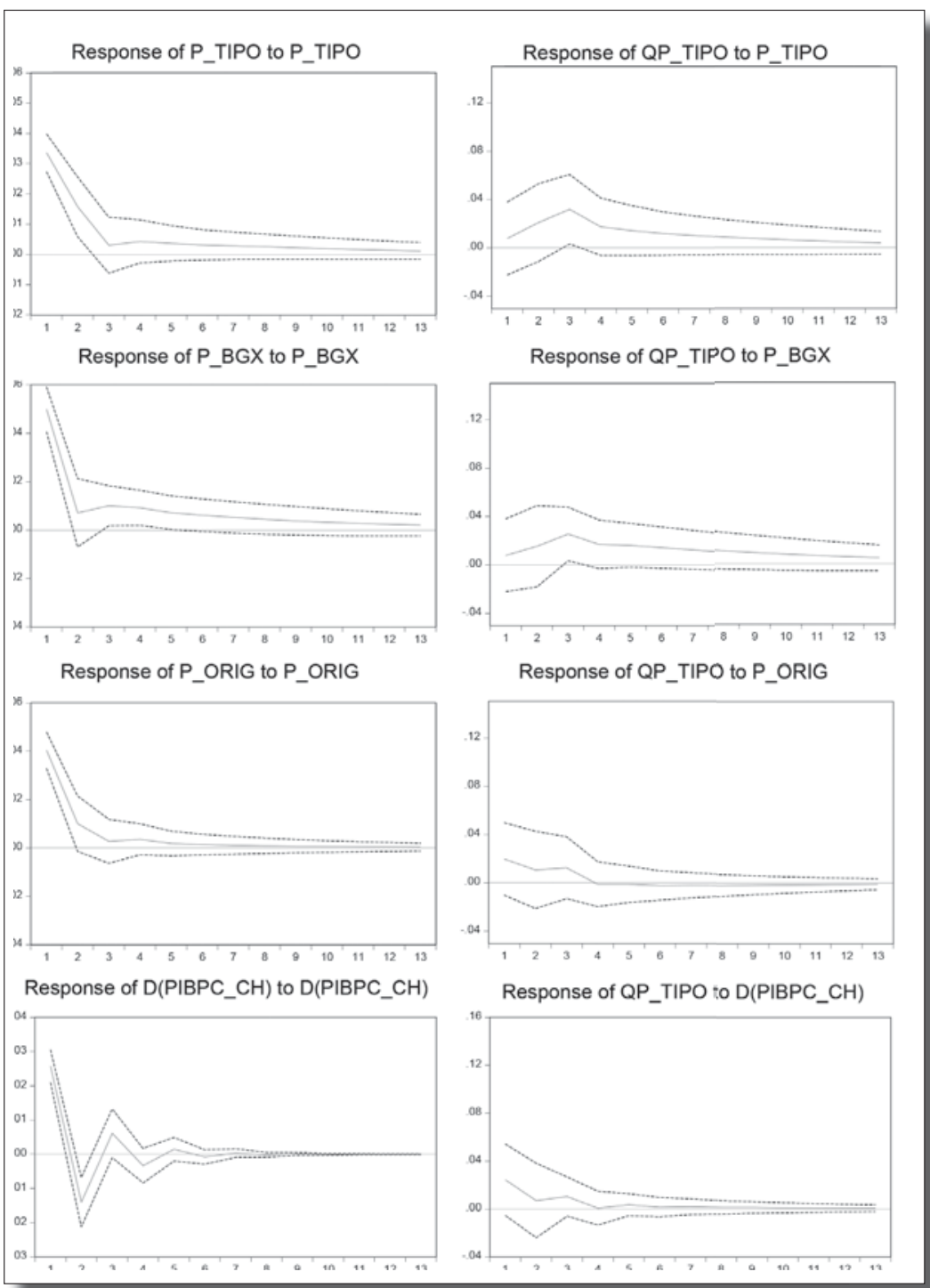

Figura 2. Respuesta al impUlso de las VARIABLES de ANÁLISIS

Fuente: elaboración propia

Por su parte, Callejón y Ortún (18) en un estudio sobre los medicamentos en España sostienen:

Incluso, la demanda puede mostrar elasticidad-precio positiva por su tendencia a desplazarse hacia los medicamentos nuevos y más caros en lo que se conoce como efecto deslizamiento, de forma que el descenso del precio relativo de los medicamentos más tradicionales, o con patente expirada, no se traduce en moderación del gasto total.

Los medicamentos se consideran bienes necesarios muy inelásticos cuya compra en la mayoría de los casos es inducida por el médico tratante, de acuerdo con su experiencia y preferencia por uno o varios laboratorios. El 
hecho, además de que el mercado comercial de los medicamentos en Colombia en el periodo de análisis sea de libre competencia y restringido a pocos laboratorios de marca, implicó un mayor poder de mercado para la fijación de precios, situación que puede llevar a este tipo de resultados. Si bien los precios tomados en este estudio son de mayorista, es claro que cualquier cambio en las condiciones de oferta y demanda se transmite a lo largo de la cadena de suministro desde el laboratorio hasta el consumidor final.

Además, la asimetría de información que se presenta en el sector de medicamentos produce diferenciales de precios entre productos que tienen un mismo principio activo. Por esta vía, de nuevo, el productor logra conseguir poder de mercado que puede manifestarse en la aplicación de una estrategia de precios que fije un margen significativo sobre los costos marginales de producción y comercialización.

Se concluye que este tipo de medicamentos de alto consumo, dada la patología que cubre, elaborado por un laboratorio de marca con un gran posicionamiento e infraestructura de comercialización, otorga a este alto poder de mercado para fijar precios, incluso como se desprende de los modelos VAR estimados, con relación neutra o positiva de la cantidad demandada ante aumentos en los precios.

Aunque la aplicación de una política farmacéutica en Colombia se oriente a incentivar el consumo de genéricos, fomentar la competencia y reducir el precio promedio de los medicamentos, hay que tener en cuenta la evidencia internacional que señala que el precio de los medicamentos de marca en general tiende a subir, y no a bajar, porque los consumidores leales son inelásticos al precio, situación que permite a los laboratorios mantener su poder de mercado. Se podría presentar así una paradoja en la que una política que incentive el uso de genéricos genere mayores precios, tanto para ellos mismos como para los de marca. En este caso los beneficios de un menor precio serían para unos pocos consumidores que migran desde marcas y similares a los genéricos.

Por último, se aclara que en los modelos desarrollados no se tuvieron en cuenta variables cualitativas que podrían incidir en el comportamiento de compra de los pacientes. Nuevas investigaciones podrían analizar su significancia o aporte para el cálculo de funciones de demanda y sus elasticidades.

\section{Referencias bibliográficas}

1. Consejo Nacional de Política Económica y Social, República de Colombia. Política farmacéutica nacional. Documento Conpes Social 155. Bogotá: Departamento Nacional de Planeación; 2012.

2. Rodríguez AS, Vásquez VJ, Gómez PK. Regulación en el mercado farmacéutico colombiano. Revista de Ciencias Sociales. 2010; 16(2):197-209.

3. Circular 03 del 2013. Comisión Nacional de Precios de Medicamentos y Dispositivos Médicos, Colombia.

4. Consejo Nacional de Política Económica y Social, Departamento Nacional de Planeación, Colombia. Documento Conpes Social. Política farmacéutica nacional. Proyecto para discusión. Bogotá: Departamento Nacional de Planeación; 2012.

5. Machado-Alba JE, Morales Plaza CD, Solarte Gómez MJ. Patrones de prescripción de antidepresivos en pacientes afiliados al Sistema General de Seguridad Social en Salud de Colombia. Rev Panam Salud Pública. 2011; 30(5):461-8.

6. Center for Global Development. A Risky business. Saving money and improving global health through better demand forecasts. Washington D. C.: Library of Congress Cataloging-in-Publication Data; 2007.

7. Managing Science for Health. MDS-3: Managing access to medicines and health technologies. Arlington, VA: Management Science for Health; 2012.

8. Ghousi R, Mehrani S, Momeni M, Anjomshoaa S. Application of data mining techniques in drug consumption forecasting help pharmaceutical industry production planning. Proceedings of the 2012 International Conference on Industrial Engineering and Operations Management; 2012 Jul 3-6; Estambul, Turquía. Irán: Iran University of Science and Technology, Narmak; 2012.

9. Ching AT. Consumer learning and heterogeneity: dynamics of demand for prescription drugs after patent expiration. Int J Ind Organ. 2010; 26(6): 619-638. 
10. Kim W, Won JH, Park S, Kang J. Demand forecasting models for medicines through wireless sensor networks data and topic trend analysis. International Journal of Distributed Sensor Networks. 2015; 36.

11. Fisher S, Cockburn I, Griliches Z, Hausman J. Characteristics of demand for pharmaceutical products: An examination of four cephalosporins. Rand J Econ.1997; 28(3):426-46.

12. Hurtado JL, Melo LF. Una metodología multivariada de desagregación temporal. Borradores de Economía del Banco de la Republica. 2010; (586):1-37.

13. Quilis EM. A matlab library of temporal disagregation methods: Summary. Madrid: Instituto Nacional de Estadística; 2004.

14. Flores de Frutos R, Pereira AM. Public capital and aggregate growth in the United States: Is public capital productive? Documentos de Trabajo del Instituto Complutense de Análisis Económico (ICAE). 1993; (13):1-33.

15. Roca O, Pereira AM. Impacto de la inversión en infraestructura sobre el producto, la ocupación y la inversión privada en España. Revista Española de Economía. 1998; 15(3):403-32.

16. Pesaran H, Shin Y. Generalized impulse response analysis in linear multivariate models. Economics Letters. 1998; 58(1):17-29.

17. Torres S, Gutiérrez JP. Mercado farmacéutico en México: tamaño, valor y concentración. Rev Panam Salud Pública. 2009; 26(1):46-50.

18. Callejón MR, Ortún RV. Los medicamentos en España: Mercado único y utilidad social. Revista Economía Industrial del Ministerio de Industria, Energía y Turismo.1990 Jul.-Agos.; 191-206. 


\section{Anexos}

\section{Anexo 1. Pruebas de estacionariedad}

Se realizan las pruebas ampliadas de Dickey y Fuller con el objetivo de verificar la presencia de raíces unitarias. Se contrasta la hipótesis nula de raíz unitaria, a efectos de establecer el orden de integración de cada variable. En la tabla adjunta se presentan los resultados de dicha prueba; los valores con asterisco indican significancia estadística al 5\%, la cual se evalúa a partir de los valores críticos de MacKinnon.

\begin{tabular}{|l|c|c|c|c|c|c|}
\hline & \multicolumn{3}{|c|}{ Niveles } & \multicolumn{3}{c|}{ Diferencias } \\
\hline Variables & Rezagos & Especificación & Estadístico & Rezagos & Especificación & Estadístico \\
\hline QP_TIPO & 1 & T y C & $-6.50^{*}$ & & & \\
\hline P_TIPO & 1 & T y C & $-6.02^{*}$ & & & \\
\hline P_ORIG & 1 & C & $-4.61^{*}$ & & & \\
\hline P_BGX & 1 & T y C & $-6.17^{*}$ & & & \\
\hline P_GX & 1 & T y C & -2.10 & 1 & N & $-10.04^{*}$ \\
\hline ING_HOG & 10 & T y C & 0.72 & 10 & T y C & $-8.22^{*}$ \\
\hline PIBCH & 4 & T y C & -1.21 & 3 & C & $-8.17^{*}$ \\
\hline IPPT & 1 & T y C & -2.33 & 1 & N & $-4.98^{*}$ \\
\hline TRM & 1 & T y C & -3.07 & 1 & N & $-4.83^{*}$ \\
\hline
\end{tabular}

T, C y N indican tendencia, constante y sin los componentes anteriores, respectivamente.

*Significancia al $5 \%$.

Fuente: elaboración propia

Como se observa, las variables $\mathrm{I}(0)$ o estacionarias fueron: cantidad demandada per cápita del producto "tipo" (QP_TIPO), precio del producto "tipo" (P_TIPO), precio originales (P_ORIG) y precio genéricos de marca (P_BGX). Las variables I(1) o no estacionarias fueron: precio de genéricos (P_GX), ingreso de los hogares (ING_HOG), PIB método de desagregación de Chow y Lin (PIBCH), el índice de precios al productor total (IPPT) y la tasa representativa del mercado (TRM). Por tal razón las anteriores variables se incluyen en el modelo en primeras diferencias.

\section{Anexo 2. Selección de la variable proxy del ingreso a partir de la estimación de los modelos VAR}

Se proponen dos modelos VAR, uno con la serie desagregada de la metodología de Chow y Lin (PIBCH) y el otro con la serie del ingreso de los hogares (ING_HOG). Para la evaluación de cada modelo VAR se tuvo en cuenta el criterio de información de Hannan y Quinn 
(HQ) ${ }^{12}$ con el fin de establecer el número máximo de rezagos que era necesario incluir en el modelo. Como ejemplo, en la tabla adjunta se establece que el número de rezagos seleccionado con la variable proxy del ingreso (PIB desagregada con la metodología de Chow y Lin $[\mathrm{PIBCH}])$ es uno.

\begin{tabular}{|l|c|}
\hline Rezago & Hannan-Quinn \\
\hline 0 & -22.68 \\
\hline 1 & $-23.36^{*}$ \\
\hline 2 & -21.61 \\
\hline 3 & -20.40 \\
\hline 4 & -20.62 \\
\hline 5 & -21.73 \\
\hline
\end{tabular}

*Indica el número de rezagos que es necesario seleccionar por el criterio.

Fuente: elaboración propia

Luego de realizar el mismo procedimiento con la variable ingreso de los hogares (ING_HOG) y estimar el modelo VAR correspondiente, se concluye que el PIB con el método de desagregación de Chow y Lin (PIBCH) presenta el menor valor, según el criterio de Akaike, y por tanto es la variable más adecuada para especificar en los modelos (véase tabla adjunta).

\begin{tabular}{|l|l|}
\hline Modelo & AIC \\
\hline Ing_hog & -22.40 \\
\hline pibch & -22.91 \\
\hline
\end{tabular}

Fuente: elaboración propia.

12 Es un criterio de selección de modelos que se basa en la teoría de la información; se aplica a modelos autorregresivos y se considera consistente. Su expresión es: , donde SCE es la suma de cuadrados del error, T el tamaño muestral y $k$ el número de regresores usados en el modelo. Con este criterio el modelo resultó más parsimonioso con respecto al número de rezagos en comparación con el criterio AIC; adicionalmente, para el mismo número de rezagos el estadístico LR (razón de verosimilitud) presentó el valor más alto, lo cual ratifica la selección hecha por HannanQuinn. 


\section{Anexo 3. Modelo VAR. Cálculo de la demanda}

\begin{tabular}{|c|c|c|c|c|c|c|c|c|}
\hline & QP_TIPO & P_TPO & P_BGX & $D\left(P \_G X\right)$ & P_ORIG & D(PIBPC_CH) & D(IPPT) & D(TRM) \\
\hline$Q P$ _nPO(-1) & $\begin{array}{l}0.507112 \\
(0.11210) \\
{[4.52365]}\end{array}$ & $\begin{array}{c}0.076361 \\
(0.03208) \\
{[2.38009]}\end{array}$ & $\begin{array}{l}0.055305 \\
(0.04719) \\
{[1.17190]}\end{array}$ & $\begin{array}{c}0.005635 \\
(0.03112) \\
{[0.18111]}\end{array}$ & $\begin{array}{c}0.026519 \\
(0.03821) \\
{[0.69410]}\end{array}$ & $\begin{array}{r}-0.044417 \\
(0.02466) \\
{[-1.80146]}\end{array}$ & $\begin{array}{r}-0.657672 \\
(0.63530) \\
{[-1.03521]}\end{array}$ & $\begin{array}{r}-0.057427 \\
(0.03357) \\
{[-1.71043]}\end{array}$ \\
\hline P_TPO(-1) & $\begin{array}{c}1.113702 \\
(0.46609) \\
{[2.38945]}\end{array}$ & $\begin{array}{r}0.464610 \\
(0.13339) \\
{[3.48301]}\end{array}$ & $\begin{array}{l}0.158401 \\
(0.19622) \\
{[0.80728]}\end{array}$ & $\begin{array}{r}-0.233173 \\
(0.12937) \\
{[-1.80239]}\end{array}$ & $\begin{array}{r}-0.159005 \\
(0.15885) \\
{[-1.00095]}\end{array}$ & $\begin{array}{c}0.054972 \\
(0.10251) \\
{[0.53624]}\end{array}$ & $\begin{array}{l}1.038438 \\
(2.64143) \\
{[0.39314]}\end{array}$ & $\begin{array}{l}0.176492 \\
(0.13959) \\
{[1.26432]}\end{array}$ \\
\hline$P_{-} B G \times(-1)$ & $\begin{array}{c}0.066326 \\
(0.34996) \\
{[0.18953]}\end{array}$ & $\begin{array}{c}0.025527 \\
(0.10016) \\
{[0.25488]}\end{array}$ & $\begin{array}{l}0.187455 \\
(0.14733) \\
{[1.27238]}\end{array}$ & $\begin{array}{r}-0.110406 \\
(0.09713) \\
{[-1.13663]}\end{array}$ & $\begin{array}{r}-0.296169 \\
(0.11927) \\
{[-2.48311]}\end{array}$ & $\begin{array}{c}-0.019303 \\
(0.07697) \\
(-0.25078)\end{array}$ & $\begin{array}{c}1.356416 \\
(1.98327) \\
{[0.68393]}\end{array}$ & $\begin{array}{r}0.015499 \\
(0.10481) \\
{[0.14788]}\end{array}$ \\
\hline$D\left(P_{-} G X(-1)\right)$ & $\begin{array}{r}-0.547249 \\
(0.47958) \\
{[-1.14110]}\end{array}$ & $\begin{array}{r}0.341799 \\
(0.13725) \\
{[2.49029]}\end{array}$ & $\begin{array}{r}-0.090552 \\
(0.20189) \\
{[-0.44851]}\end{array}$ & $\begin{array}{r}-0.197910 \\
(0.13311) \\
{[-1.48679]}\end{array}$ & $\begin{array}{l}0.357908 \\
(0.16345) \\
{[2.18970]}\end{array}$ & $\begin{array}{c}0.004118 \\
(0.10548) \\
{[0.03904]}\end{array}$ & $\begin{array}{r}-0.761989 \\
(2.71786) \\
{[-0.28036 !}\end{array}$ & $\begin{array}{r}-0.145231 \\
(0.14363) \\
\mid-1.01112]\end{array}$ \\
\hline P_ORIG(-1) & $\begin{array}{r}-0.311115 \\
(0.34995) \\
{[-0.88902]}\end{array}$ & $\begin{array}{r}-0.206690 \\
(0.10015) \\
{[-2.06371]}\end{array}$ & $\begin{array}{r}-0.427904 \\
(0.14732) \\
{[-2.90452]}\end{array}$ & $\begin{array}{l}-0.126981 \\
(0.09713) \\
{[-1.30728]}\end{array}$ & $\begin{array}{l}0.349637 \\
(0.11927) \\
{[2.93143]}\end{array}$ & $\begin{array}{r}-0.127728 \\
(0.07697) \\
{[-1.65948]}\end{array}$ & $\begin{array}{r}-0.110099 \\
(1.98324) \\
{[-0.05551]}\end{array}$ & $\begin{array}{l}0.046788 \\
(0.10481) \\
{[0.44640]}\end{array}$ \\
\hline $\mathrm{D}(\mathrm{PIBPC} \mathrm{CH} \mathrm{CH}(-1))$ & $\begin{array}{r}-0.210275 \\
(0.58785) \\
{[-0.35770]}\end{array}$ & $\begin{array}{r}-0.196362 \\
(0.16824) \\
{[-1.16716]}\end{array}$ & $\begin{array}{r}-0.053812 \\
(0.24747) \\
{[-0.21744]}\end{array}$ & $\begin{array}{r}-0.189724 \\
(0.16316) \\
{[-1.16278]}\end{array}$ & $\begin{array}{r}-0.091078 \\
(0.20035) \\
{[-0.45458]}\end{array}$ & $\begin{array}{r}-0.439971 \\
(0.12929) \\
{[-3.40292]}\end{array}$ & $\begin{array}{r}-4.526771 \\
(3.33144) \\
{[-1.35880]}\end{array}$ & $\begin{array}{r}-0.046625 \\
(0.17606) \\
{[-0.26482]}\end{array}$ \\
\hline D(IPPT(-1)) & $\begin{array}{r}-0.005359 \\
(0.02601) \\
{[-0.20604]}\end{array}$ & $\begin{array}{r}-0.002708 \\
(0.00744) \\
{[-0.36384]}\end{array}$ & $\begin{array}{l}0.006594 \\
(0.01095) \\
{[0.60220]}\end{array}$ & $\begin{array}{r}-0.000100 \\
(0.00722) \\
{[-0.01386]}\end{array}$ & $\begin{array}{r}-0.006323 \\
(0.00886) \\
{[-0.71329]}\end{array}$ & $\begin{array}{r}-0.007549 \\
(0.00572) \\
{[-1.31974]}\end{array}$ & $\begin{array}{r}0.294030 \\
(0.14739) \\
{[1.99485]}\end{array}$ & $\begin{array}{r}-0.015288 \\
(0.00779) \\
{[-1.96262]}\end{array}$ \\
\hline D(TRM(-1)) & $\begin{array}{r}-0.009260 \\
(0.50472) \\
(-0.01835)\end{array}$ & $\begin{array}{c}0.008543 \\
(0.14445) \\
{[0.05914]}\end{array}$ & $\begin{array}{r}-0.000522 \\
(0.21248) \\
{[-0.00246]}\end{array}$ & $\begin{array}{r}-0.125297 \\
(0.14009) \\
(-0.89439)\end{array}$ & $\begin{array}{r}-0.012651 \\
(0.17202) \\
{[-0.07354]}\end{array}$ & $\begin{array}{c}0.041388 \\
(0.11101) \\
{[0.37283]}\end{array}$ & $\begin{array}{l}4.523346 \\
(2.86034) \\
{[1.58140]}\end{array}$ & $\begin{array}{l}0.551973 \\
(0.15116) \\
{[3.65149]}\end{array}$ \\
\hline c & $\begin{array}{r}-5.098801 \\
(2.65817) \\
{[-1.91816]}\end{array}$ & $\begin{array}{r}3.642715 \\
(0.76075) \\
{[4.78830]}\end{array}$ & $\begin{array}{l}5.334487 \\
(1.11904) \\
{[4.76702]}\end{array}$ & $\begin{array}{l}2.240311 \\
(0.73781) \\
{[3.03645]}\end{array}$ & $\begin{array}{l}5.599142 \\
(0.90596) \\
{[6.18032]}\end{array}$ & $\begin{array}{c}0.396911 \\
(0.58464) \\
{[0.67890]}\end{array}$ & $\begin{array}{r}-12.01942 \\
(15.0643) \\
{[-0.79787]}\end{array}$ & $\begin{array}{r}-1.289173 \\
(0.79612) \\
{[-1.61932]}\end{array}$ \\
\hline $\begin{array}{l}\text { R-squared } \\
\text { Adj R-squared } \\
\text { Sum sq. resids } \\
\text { SE. equastion } \\
\text { F-statistic } \\
\text { Log likelihood } \\
\text { Akaike AC } \\
\text { Schwarz SC } \\
\text { Mean dependent } \\
\text { S.D. dependent }\end{array}$ & $\begin{array}{l}0.596905 \\
0.531094 \\
0.676489 \\
0.117499 \\
9.069948 \\
46.78873 \\
-1.303060 \\
-0.983336 \\
-2.885738 \\
0.171589\end{array}$ & $\begin{array}{l}0.573452 \\
0.503812 \\
0.055409 \\
0.033627 \\
8.234473 \\
119.3516 \\
-3.805228 \\
-3.485504 \\
4.531737 \\
0.047739\end{array}$ & $\begin{array}{l}0.361492 \\
0.257246 \\
0.119891 \\
0.049465 \\
3.467680 \\
96.96845 \\
-3.033395 \\
-2.713671 \\
4.427231 \\
0.057395\end{array}$ & $\begin{array}{l}0.313402 \\
0.201304 \\
0.052117 \\
0.032613 \\
2.795793 \\
121.1281 \\
-3.866487 \\
-3.546763 \\
-0.002264 \\
0.036492\end{array}$ & $\begin{array}{l}0.427921 \\
0.334520 \\
0.078581 \\
0.040046 \\
4.581565 \\
109.2197 \\
-3.455850 \\
-3.136126 \\
5.365653 \\
0.049090\end{array}$ & $\begin{array}{l}0.332209 \\
0.223182 \\
0.032725 \\
0.025843 \\
3.047032 \\
134.6236 \\
-4.331848 \\
-4.012125 \\
0.002213 \\
0.029321\end{array}$ & $\begin{array}{l}0.273715 \\
0.155138 \\
21.72672 \\
0.665885 \\
2.308332 \\
-53.82333 \\
2.166322 \\
2.486046 \\
-0.073095 \\
0.724446\end{array}$ & $\begin{array}{l}0.305876 \\
0.192549 \\
0.060681 \\
0.035191 \\
2.699069 \\
116.7160 \\
-3.714346 \\
-3.394622 \\
-0.006204 \\
0.039162\end{array}$ \\
\hline \multicolumn{2}{|c|}{$\begin{array}{l}\text { Determinant resid covariance } \\
\text { Log likelihood }\end{array}$} & $\begin{array}{r}4.96 E-21 \\
1.29 E-21 \\
736.5632 \\
-22.91597 \\
-20.35818\end{array}$ & & & & & & \\
\hline
\end{tabular}

Fuente: elaboración propia

\section{Anexo 4. Prueba de validación del modelo VAR}

Resultados del test de Portmanteau ${ }^{13}$

\begin{tabular}{|l|l|l|l|l|l|}
\hline Rezago & Q-stat & Prob. & Adj Q-Stat & Prob. & gl \\
\hline 1 & 24.94 & NA* & 25.38 & NA $^{*}$ & NA* \\
\hline 2 & 71.89 & 0.23 & 74 & 0.18 & 64 \\
\hline 3 & 122.11 & 0.63 & 126.96 & 0.51 & 128 \\
\hline 4 & 178.67 & 0.75 & 187.71 & 0.57 & 192 \\
\hline 5 & 228.91 & 0.89 & 242.69 & 0.71 & 256 \\
\hline 6 & 287.4 & 0.90 & 307.93 & 0.68 & 320 \\
\hline
\end{tabular}

Fuente: elaboración propia

El análisis del valor de probabilidad (prob.) para los seis rezagos muestra porcentajes muy superiores al 5\%, con lo cual no se rechaza la hipótesis nula de ruido blanco para las perturbaciones.

$13{ }^{13}$ Este test se utiliza, entre otras oportunidades, en modelos VAR para verificar el comportamiento ruido blanco de 295 los residuos. 
Prueba de estabilidad: invertibilidad y estacionariedad (inverso de las raíces del polinomio de rezago)

\begin{tabular}{|c|c|c|}
\hline Raíz número & Valor del inverso de la raíz & Módulo del inverso de la raíz \\
\hline 1 & 0.89 & 0.89 \\
\hline 2 & 0.55 & 0.55 \\
\hline 3 & $0.44-0.23 \mathrm{i}$ & 0.50 \\
\hline 4 & $0.44+0.23 \mathrm{i}$ & 0.50 \\
\hline 5 & -0.48 & 0.48 \\
\hline 6 & $-0.11-0.25 \mathrm{i}$ & 0.27 \\
\hline 7 & $-0.11+25 \mathrm{i}$ & 0.27 \\
\hline 8 & 0.09 & 0.09 \\
\hline
\end{tabular}

Fuente: elaboración propia

Se concluye que todas las raíces se encuentran dentro del círculo unitario.

\section{Anexo 5. Respuesta de QP_TIPO ante el impulso de las demás variables del modelo}

\begin{tabular}{|l|c|c|c|c|c|c|c|c|}
\hline Periodo & QP_TIPO & P_TIPO & P_BGX $_{-}$ & D(P_GX) & P_ORIG & D(PIBPC_CH) & D(IPPT) & D(TRM) \\
\hline $\mathrm{t}$ & 0.12 & 0.01 & 0.01 & 0.02 & 0.02 & 0.03 & 0.00 & -0.02 \\
\hline $\mathrm{t}+1$ & 0.06 & 0.03 & 0.02 & 0.01 & 0.01 & 0.01 & -0.01 & -0.01 \\
\hline $\mathrm{t}+2$ & 0.04 & $\mathbf{0 . 0 4}$ & 0.03 & 0.03 & 0.01 & 0.02 & -0.01 & -0.01 \\
\hline $\mathrm{t}+3$ & 0.03 & $\mathbf{0 . 0 3}$ & 0.02 & 0.02 & 0.00 & 0.01 & -0.01 & -0.01 \\
\hline $\mathrm{t}+4$ & 0.03 & $\mathbf{0 . 0 3}$ & 0.02 & 0.01 & 0.00 & 0.01 & -0.01 & -0.01 \\
\hline $\mathrm{t}+5$ & 0.02 & $\mathbf{0 . 0 2}$ & 0.02 & 0.01 & 0.00 & 0.00 & 0.00 & -0.01 \\
\hline
\end{tabular}

Fuente: elaboración propia

\section{Anexo 6. Valores de probabilidad del test de la traza para las diferentes opciones de cointegración}

\begin{tabular}{|l|c|c|c|c|}
\hline \multirow{2}{*}{ Ho: número máximo de ecuaciones de cointegración } & \multicolumn{4}{|c|}{ Opciones de relaciones de cointegración } \\
\cline { 2 - 5 } & a) & b) & c) & d) \\
\hline $\mathrm{r}=0$ & 0.05 & 0.03 & 0.01 & 0.00 \\
\hline $\mathrm{r}=1$ & $\mathbf{0 . 1 2}$ & 0.11 & 0.07 & 0.05 \\
\hline $\mathrm{r}=2$ & 0.23 & 0.20 & 0.07 & 0.26 \\
\hline $\mathrm{r}=3$ & 0.48 & 0.25 & 0.02 & 0.53 \\
\hline
\end{tabular}

a) Ssin tendencia determinista en niveles y sin intercepto en la ecuación de cointegración;

b) sin tendencia determinista en niveles y con intercepto en la ecuación de cointegración; c) con tendencia determinista en niveles y con intercepto en la ecuación de cointegración; y d) con tendencia determinista en niveles y 296 con tendencia e intercepto en la ecuación de cointegración.

Fuente: elaboración propia 


\section{Anexo 7. Modelo VAR con corrección de error}

\begin{tabular}{|c|c|c|c|c|c|c|c|c|}
\hline & QP_TIPO & P_TIPO & P_BGX & D(P_GX) & P_ORIG & D(PIBPC_CH) & D(PPT) & D(TRM) \\
\hline$Q P_{-} T P Q(-1)$ & $\begin{array}{l}0.418006 \\
(0.12003) \\
{[3.48264]}\end{array}$ & $\begin{array}{l}0.062098 \\
(0.03515) \\
{[1.76648]}\end{array}$ & $\begin{array}{l}0.058614 \\
(0.05223) \\
{[1.12233]}\end{array}$ & $\begin{array}{l}0.003446 \\
(0.03443) \\
{[0.10007]}\end{array}$ & $\begin{array}{l}0.026752 \\
(0.04229) \\
{[0.63255]}\end{array}$ & $\begin{array}{r}-0.057373 \\
(0.02691) \\
{[-2.13243]}\end{array}$ & $\begin{array}{r}-0.545651 \\
(0.70211) \\
{[-0.77716]}\end{array}$ & $\begin{array}{r}-0.013223 \\
(0.03372) \\
\mid-0.39211)\end{array}$ \\
\hline P_TIPO(-1) & $\begin{array}{l}0.903247 \\
(0.47000) \\
{[1.92182]}\end{array}$ & $\begin{array}{r}0.430924 \\
(0.13765) \\
{[3.13048]}\end{array}$ & $\begin{array}{l}0.166215 \\
(0.20450) \\
{[0.81277]}\end{array}$ & $\begin{array}{r}-0.238345 \\
(0.13483) \\
{[-1.76771]}\end{array}$ & $\begin{array}{r}-0.158456 \\
(0.16560) \\
{[-0.95683]}\end{array}$ & $\begin{array}{r}0.024370 \\
(0.10535) \\
{[0.23131]}\end{array}$ & $\begin{array}{l}1.303014 \\
(2.74931) \\
{[0.47394]}\end{array}$ & $\begin{array}{r}0.280896 \\
(0.13205) \\
{[2.12724]}\end{array}$ \\
\hline P_BGX(-1) & $\begin{array}{l}0.106623 \\
(0.34273) \\
{[0.31110]}\end{array}$ & $\begin{array}{l}0.031977 \\
(0.10038) \\
{[0.31856]}\end{array}$ & $\begin{array}{l}0.185959 \\
(0.14913) \\
{[1.24698]}\end{array}$ & $\begin{array}{r}-0.109416 \\
(0.09832) \\
{[-1.11282]}\end{array}$ & $\begin{array}{r}-0.296274 \\
(0.12076) \\
(-2.45337]\end{array}$ & $\begin{array}{r}-0.013443 \\
(0.07683) \\
(-0.17498]\end{array}$ & $\begin{array}{l}1.305756 \\
(2.00485) \\
{[0.65130]}\end{array}$ & $\begin{array}{r}-0.004492 \\
(0.09629) \\
(-0.04665]\end{array}$ \\
\hline$D\left(P_{-} G X(-1)\right)$ & $\begin{array}{r}-0.567133 \\
(0.46882) \\
{[-1.20971]}\end{array}$ & $\begin{array}{l}0.338616 \\
(0.13731) \\
{[2.46609]}\end{array}$ & $\begin{array}{r}-0.089813 \\
(0.20399) \\
{[-0.44028]}\end{array}$ & $\begin{array}{r}-0.198398 \\
(0.13449) \\
{[-1.47514]}\end{array}$ & $\begin{array}{l}0.357959 \\
(0.16519) \\
{[2.16696]}\end{array}$ & $\begin{array}{l}0.001227 \\
(0.10509) \\
{[0.01167]}\end{array}$ & $\begin{array}{l}-0.736992 \\
(2.74241) \\
{[-0.26874]}\end{array}$ & $\begin{array}{r}-0.135366 \\
(0.13172) \\
{[-1.02771]}\end{array}$ \\
\hline P_ORIG(-1) & $\begin{array}{r}-0.241611 \\
(0.34414) \\
{[-0.70208]}\end{array}$ & $\begin{array}{r}-0.195565 \\
(0.10079) \\
-1.94027]\end{array}$ & $\begin{array}{r}-0.430485 \\
(0.14974) \\
{[-2.87488]}\end{array}$ & $\begin{array}{r}-0.125273 \\
(0.09873) \\
-1.26889]\end{array}$ & $\begin{array}{r}0.349456 \\
(0.12126) \\
{[2.88191]}\end{array}$ & $\begin{array}{r}-0.117622 \\
(0.07714) \\
{[-1.52474]}\end{array}$ & $\begin{array}{r}-0.197477 \\
(2.01309) \\
{[-0.09810)}\end{array}$ & $\begin{array}{c}0.012308 \\
(0.09669) \\
{[0.127301}\end{array}$ \\
\hline D(PIBPC_CH(-1)) & $\begin{array}{r}-0.225431 \\
(0.57456) \\
(-0.39235]\end{array}$ & $\begin{array}{r}-0.198788 \\
(0.16828) \\
{[-1.18129]}\end{array}$ & $\begin{array}{r}-0.053249 \\
(0.25000) \\
{[-0.21299]}\end{array}$ & $\begin{array}{r}-0.190097 \\
(0.16483) \\
-1.15329]\end{array}$ & $\begin{array}{r}-0.091036 \\
(0.20245) \\
{[-0.44968]}\end{array}$ & $\begin{array}{r}-0.442175 \\
(0.12879) \\
{[-3.43319]}\end{array}$ & $\begin{array}{r}4.507718 \\
(3.36098) \\
\mid-1.34119\end{array}$ & $\begin{array}{r}-0.039106 \\
(0.16143) \\
1-0.24226]\end{array}$ \\
\hline D(IPPT $(-1))$ & $\begin{array}{l}0.012304 \\
(0.02721) \\
{[0.45216]}\end{array}$ & $\begin{array}{l}0.000119 \\
(0.00797) \\
{[0.01492]}\end{array}$ & $\begin{array}{l}0.005938 \\
(0.01184) \\
{[0.50147]}\end{array}$ & $\begin{array}{l}0.000334 \\
(0.00781) \\
{[0.04278]}\end{array}$ & $\begin{array}{r}-0.006369 \\
(0.00959) \\
{[-0.66422]}\end{array}$ & $\begin{array}{r}-0.004981 \\
(0.00610) \\
{[-0.81656]}\end{array}$ & $\begin{array}{l}0.271825 \\
(0.15918) \\
{[1.70763]}\end{array}$ & $\begin{array}{r}-0.024050 \\
(0.00765) \\
\mid-3.14570]\end{array}$ \\
\hline D(TRM $(-1))$ & $\begin{array}{r}-0.162085 \\
(0.50038) \\
{[-0.32393]}\end{array}$ & $\begin{array}{r}-0.015918 \\
(0.14655) \\
{[-0,10862]}\end{array}$ & $\begin{array}{l}0.005153 \\
(0.21772) \\
{[0.02367]}\end{array}$ & $\begin{array}{r}-0.129052 \\
(0.14355) \\
{[-0.89901]}\end{array}$ & $\begin{array}{r}-0.012253 \\
(0.17631) \\
{[-0.06950]}\end{array}$ & $\begin{array}{c}0.019166 \\
(0.11216) \\
{[0.17087]}\end{array}$ & $\begin{array}{l}4.715472 \\
(292702) \\
{[1.61101]}\end{array}$ & $\begin{array}{l}0.627787 \\
(0.14058) \\
{[4.46560)}\end{array}$ \\
\hline c & $\begin{array}{r}-4969871 \\
(2.59878) \\
{[-1.91239]}\end{array}$ & $\begin{array}{l}3.663352 \\
(0.76114) \\
{[4.81298]}\end{array}$ & $\begin{array}{l}5.329700 \\
(1.13077) \\
{[4.71332]}\end{array}$ & $\begin{array}{l}2.243479 \\
(0.74554) \\
(3.00920)\end{array}$ & $\begin{array}{c}5.593806 \\
(0.91569) \\
{[6.11430]}\end{array}$ & $\begin{array}{l}0.415659 \\
(0.58255) \\
{[0.71352]}\end{array}$ & $\begin{array}{r}-12.18150 \\
(15.2020) \\
{[-0.80131]}\end{array}$ & $\begin{array}{r}-1.353134 \\
(0.73014) \\
-1.85325\end{array}$ \\
\hline COINTEO03 & $\begin{array}{l}0.158141 \\
(0.08701) \\
{[1.81756]}\end{array}$ & $\begin{array}{c}0.025312 \\
(0.02548) \\
{[0.99330]}\end{array}$ & $\begin{array}{r}-0.005872 \\
(0.03786) \\
(-0.15510]\end{array}$ & $\begin{array}{l}0.003886 \\
(0.02496) \\
{[0.15568]}\end{array}$ & $\begin{array}{r}-0.000412 \\
(0.03066) \\
{[-0.01344]}\end{array}$ & $\begin{array}{l}0.022995 \\
(0.01950) \\
{[1.17901]}\end{array}$ & $\begin{array}{r}-0.198809 \\
(0.50896) \\
{[-0.39061]}\end{array}$ & $\begin{array}{r}-0.078452 \\
(0.02445) \\
{[-3.20929]}\end{array}$ \\
\hline $\begin{array}{l}\text { R-squared } \\
\text { Ad). R-squared } \\
\text { Sum sq. resids } \\
\text { S.E. equation } \\
\text { F-statstic } \\
\text { Log likelihood } \\
\text { Akaike AIC } \\
\text { Schwar SC } \\
\text { Mean dependent } \\
\text { S.D. dependent }\end{array}$ & $\begin{array}{l}0.622861 \\
0.552148 \\
0.632929 \\
0.114830 \\
8.908238 \\
48.71891 \\
-1.335135 \\
-0.979886 \\
-2.885738 \\
0.171589\end{array}$ & $\begin{array}{l}0.582043 \\
0.503677 \\
0.054293 \\
0.033632 \\
7.427164 \\
119.9417 \\
-3.791092 \\
-3.435843 \\
4.531737 \\
0.047739\end{array}$ & $\begin{array}{l}0.361812 \\
0.242152 \\
0.119831 \\
0.049965 \\
3.023663 \\
96.98298 \\
-2.999413 \\
-2.644164 \\
4.427231 \\
0.057395\end{array}$ & $\begin{array}{r}0.313748 \\
0.185076 \\
0.052091 \\
0.032943 \\
2.438354 \\
121.1428 \\
-3.832509 \\
-3.477260 \\
-0.002264 \\
0.036492\end{array}$ & $\begin{array}{r}0.427923 \\
0.320659 \\
0.078580 \\
0.040461 \\
3.989425 \\
109.2198 \\
-3.421371 \\
-3.066122 \\
5.365653 \\
0.049090\end{array}$ & $\begin{array}{l}0.351004 \\
0.229317 \\
0.031804 \\
0.025741 \\
2.884485 \\
135.4515 \\
4.325114 \\
-3.9701655 \\
0.002313 \\
0.0291321\end{array}$ & $\begin{array}{l}0.276017 \\
0.140270 \\
21.65788 \\
0.671719 \\
2.033318 \\
-53.73130 \\
2.197631 \\
2.552880 \\
-0.073095 \\
0.724446\end{array}$ & $\begin{array}{r}0.428504 \\
0.321348 \\
0.049961 \\
0.032262 \\
3.998896 \\
1223534 \\
-3.874256 \\
-3.519007 \\
-0.006204 \\
0.039162\end{array}$ \\
\hline \multicolumn{2}{|c|}{$\begin{array}{l}\text { Determinant resid covariance (dof adj) } \\
\text { Determinant resid covariance } \\
\text { Log likelithood } \\
\text { Akaike information criterion } \\
\text { Schwar criterion }\end{array}$} & $\begin{array}{r}3.96 E-21 \\
8.72 E-22 \\
747.8556 \\
-23.02950 \\
-20.18751\end{array}$ & & & & & & \\
\hline
\end{tabular}

Fuente: elaboración propia

\section{Anexo 8. Test de Portmanteau e inverso de las raíces} del polinomio característico. VAR con corrección de error

\begin{tabular}{|c|c|c|c|c|c|}
\hline Lags & Q-Stat & Prob. & Adj Q-Stat & Prob. & df \\
\hline 1 & 23.23 & NA $^{*}$ & 23.64 & NA $^{*}$ & NA $^{*}$ \\
\hline 2 & 70.15 & 0.28 & 72.24 & 0.22 & 64 \\
\hline 3 & 126.46 & 0.52 & 131.61 & 0.40 & 128 \\
\hline 4 & 174.71 & 0.81 & 183.44 & 0.66 & 192 \\
\hline 5 & 223.95 & 0.93 & 237.32 & 0.79 & 256 \\
\hline 6 & 281.32 & 0.94 & 301.32 & 0.77 & 320 \\
\hline 7 & 353.14 & 0.87 & 383.00 & 0.50 & 384 \\
\hline 8 & 409.93 & 0.90 & 448.86 & 0.48 & 448 \\
\hline
\end{tabular}

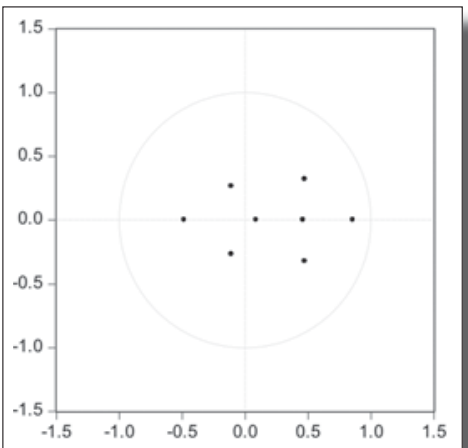

Fuente: elaboración propia 